\title{
Finite Difference Schemes for Black-Scholes with Asian Option
}

\author{
Wei Zhao ${ }^{1,2}$ \\ ${ }^{1}$ School of Economics and Resource Management, Beijing Normal University, Beijing, China \\ ${ }^{2}$ Chairman Office, Agriculture bank of china, Beijing, China \\ Correspondence: Wei Zhao, School of Economics and Resource Management, Beijing Normal University, Beijing, \\ China.
}

Received: November 15, 2016

doi:10.11114/aef.v4i1.2098

\author{
Accepted: December 5, 2016 \\ Available online: December 22, 2016 \\ URL: http://dx.doi.org/10.11114/aef.v4i1.2098
}

\begin{abstract}
Asian option is quite different from European option which can be exercised on the date of expiration, and not like the American option which exercised anytime during the period of contract. It is an unusual derivative that the option payoff depends on the average of a stock price or underlying asset over a certain period in the future. There are some demands from real world application. The attractive point is that Asian option is cheaper than the other options to hedge the similar risk. In addition, some investors need security which can protect them from the volatility and risk from the market, at the same time, the average option can provide an investor with a greater level of flexibility to make a deal. This paper focus on the point that using Finite-difference method to solve the Black-Scholes partial differential equation for the Asian Option valuation problem.
\end{abstract}

Keywords: Asian Option; finite-difference methods, numerical solutions, black scholes

\section{The Black-Scholes and Finite-difference Method}

Finite-difference approaches which are very powerful and flexible are a kind of method that give numerical answer to the partial differential equations. Once the Black-Scholes equation is reduced to the diffusion equation it is a relatively simple matter to find exact solution. This is, of course, because the diffusion equation is a far simpler and less cluttered equation than the Black-Scholes equation. For precisely this reason also, it would be easier to find numerical solution than to solve the Black-Scholes equation directly.

In fact, under some circumstance there are no way to reduce the problem to a constant coefficient diffusion equation. For example, we cannot easily deal multifactor model in one or more dimensions with the method which applied to Black-Scholes equation. Things always have two sides, solution to partial differential equation by converting it to finite-difference equation does not sound well. In some situation, the result of these schemes is not accurate and the effect which applied to parameter often failed its object. To the end, we must discuss some disadvantage of numerical method and some problem we might meet.

\subsection{Accuracy and Order}

There are two kinds of error in finite difference approach, one is round-off error which lose the precision because of computing rounding, another one is truncation error which is the difference between the solution of differential equation and the approximation. In other words, the truncation error arise is made during the approximation procedure due to the "finite" number of time steps. Even under the condition of "infinite" time steps, the truncation error still exist because of the Taylor expansion.

Normally, the main interests we focus is the local truncation error of an approach, which expressed by using Big - $O$ notation, "local" means the error refers to the error from the application of the approach. For it is convenient to proceed, we take the reminder term of a Taylor polynomial for the local truncation error. The Taylor polynomial for $f\left(x_{0}+h\right)$, which is

where $\mathrm{x}_{0}<\xi<\mathrm{x}_{0}+\mathrm{h}$,

$$
R_{n}\left(x_{0}+h\right)=\frac{f^{n+1}(\xi)}{(n+1) !}(h)^{n+1}
$$

taking the forward-difference formula for the first derivative $f\left(x_{i}\right)=f\left(x_{0}+i h\right)$, 


$$
f\left(x_{0}+i h\right)=f\left(x_{0}\right)+f^{\prime}\left(x_{0}\right) i h+\frac{f^{\prime \prime}(\xi)}{2 !}(i h)^{2}
$$

and this leads to

$$
\frac{f\left(x_{0}+i h\right)-f\left(x_{0}\right)}{i h}=f^{\prime}\left(x_{0}\right)+\frac{f^{\prime \prime}(\xi)}{2 !} i h
$$

and the value on the left is the approximation from the finite difference approach and the value on the right is the value of interest plus a remainder. The local truncation error is just the remainder. It can be showed below:

$$
\frac{f\left(x_{0}+i h\right)-f\left(x_{0}\right)}{i h}=f^{\prime}\left(x_{0}\right)+O(h)
$$

Analyzing the equation, we can find that the local truncation error is proportional to the step sizes.

\subsection{Computational Cost}

The computational methods have always a trade-off between speed and accuracy, i.e. between maximizing speed and minimizing errors. To gain a better accuracy, one must incur a cost of lengthier computing time. Fortunately, some methods converge to the correct solution faster than other methods, so a desired accuracy can be obtained with fewer computer operations.

\section{Simplified Explicit Scheme for Asian Option}

we will construct a Simplified explicit scheme for Asian option which is modeled by equation. First step we begin with the step grid and construct a mesh web. We introduce a discrete grid with steps $\Delta t=\mathrm{k}, \Delta R=\mathrm{h}$, where $\Delta \mathrm{R}$ is the grid step for the stock price, and $\Delta \mathrm{t}$ is the grid step for the time, and set

$$
H_{n}^{m}=H(m \Delta t, n \Delta R)
$$

All the difference schemes involve a parameter that is given by $\alpha=\Delta t /\left(\Delta R^{\wedge} 2\right)$, Consider the Black-Scholes problem for the Asian option

$$
\frac{\partial H}{\partial t}+\frac{1}{2} \sigma^{2} R^{2} \frac{\partial^{2} H}{\partial R^{2}}+(1-r R) \frac{\partial H}{\partial R}=0
$$

Divide the life of the option into $\mathrm{M}$ time steps of length

$$
\Delta \mathrm{t}=\frac{\mathrm{T}}{\mathrm{M}}
$$

Divide the $[0, \mathrm{R}]$ into $\mathrm{N}$ intervals of length

$$
\Delta R=\frac{R}{N}
$$

and write

$$
H_{n}^{m}=H(n \Delta R)(m \Delta t)
$$

Where $\mathrm{n}=0,1,2, \mathrm{~N}, \mathrm{~m}=0,1,2, \mathrm{M}$.

\subsection{Finite-Difference Approximations}

Using Taylor's theorem, we can approximate

$$
\begin{gathered}
\frac{\partial H}{\partial t}=\frac{H_{n}^{m+1}-H_{n}^{m}}{\Delta t}+O(\Delta t) \\
\frac{\partial H}{\partial R}=\frac{H_{n+1}^{m+1}-H_{n}^{m+1}}{\Delta R}+O(\Delta R) \\
\frac{\partial^{2} H}{\partial R^{2}}=\frac{H_{n-1}^{m+1}+H_{n+1}^{m+1}-2 H_{n}^{m+1}}{(\Delta R)^{2}}+O\left(\Delta R^{2}\right)
\end{gathered}
$$

There are some non-constant coefficient problems. Note, for future reference, that $\mathrm{t}=\mathrm{m} \Delta \mathrm{t}$ and $\mathrm{R}=\mathrm{n} \Delta \mathrm{R}$ we have

$$
R \frac{\partial H}{\partial R}=n\left(H_{n+1}^{m+1}-H_{n}^{m+1}\right)+O\left(\Delta R^{2}\right)
$$




$$
R^{2} \frac{\partial^{2} H}{\partial R^{2}}=n^{2}\left(H_{n-1}^{m+1}+H_{n+1}^{m+1}-2 H_{n}^{m+1}\right)+O\left(\Delta R^{4}\right)
$$

To the end, we substitute the explicit finite-difference approximations into the Black-Scholes equation gives the linear system

$$
H_{n}^{m}=A_{n} * H_{n}^{m+1}+B_{n} * H_{n+1}^{m+1}+C_{n} * H_{n-1}^{m+1}
$$

Where

$$
\begin{gathered}
A_{n}=1-\left(\delta^{2} n^{2}+\frac{1}{\Delta R}-r n\right) \Delta t \\
B_{n}=\left(\frac{1}{2} \delta^{2} n^{2}+\frac{1}{\Delta R}-r n\right) \Delta t \\
C_{n}=\frac{1}{2} \delta^{2} n^{2} \Delta t
\end{gathered}
$$

and this expression is accurate to $O\left(\Delta R^{2}, \Delta t\right)$

\section{Algorithm Execution}

After we finished all the mathematics module, we could use Mathematica notebook to simulate the solution. we need to translate the equation to Mathematica notebook language.

Therefore, we set $r$ as interest rate, $\sigma$ is the diffusion parameter and $T$ is the expiry day. ' $R$ ' is set to Rmax which we fixed it as limited constant.

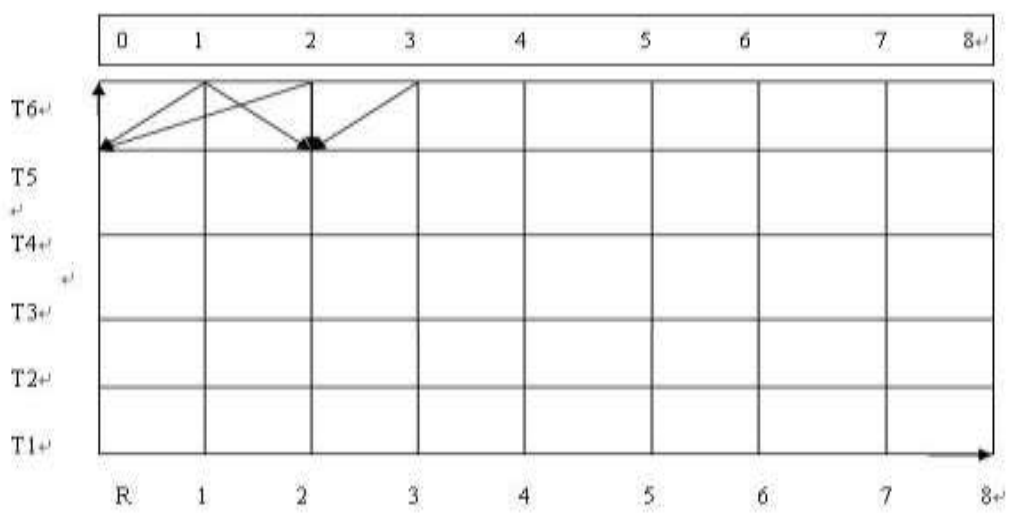

The graphic above show that how the computation procedure should go, First, we get the condition payoff:

$$
H\left(R_{T}, T\right)=\left(1-\frac{1}{T} R_{T}\right)^{+}
$$

The payoff represents the points on the upside of the rectangle. And from the condition:

$$
H(R, t)=0 \quad R \rightarrow \infty
$$

We know all the value on the right side of the rectangle is zero. For the boundary condition:

$$
\frac{\partial H}{\partial t}+\frac{\partial H}{\partial R}=0 \quad R \rightarrow 0
$$

We use the finite difference method

$$
\frac{H_{0 v}-H_{0 v-1}}{\Delta t}+\frac{-3 H_{0 v}+4 H_{1 v}-H_{2 v}}{2 \Delta R}=0
$$

to compute the $H_{0 v-1}$, then we can get the value on left side of the rectangle. Then according to the equation:

$$
H_{n}^{m}=A_{n} * H_{n}^{m+1}+B_{n} * H_{n+1}^{m+1}+C_{n} * H_{n-1}^{m+1}
$$

From the backward way, we use three known point to compute one unknown point and finally compute all of the point of the matrix. 
Using Mathematica notebook, we set a series parameter and see the performance as below:

hnought $\left[R \_\right]:=\operatorname{Max}[1-R / T, 0.0]$

$\mathrm{r}=0.05 ;$ sigma $=0.4 ; \mathrm{T}=1 ; \operatorname{Rmax}=8$;

time steps $=4000$; spatialsteps $=150$;

$\mathrm{dt}=\mathrm{T} /$ timesteps $; \mathrm{dR}=\mathrm{Rmax} /$ spatialsteps $; \mathrm{dtoR}=\mathrm{dt} / \mathrm{dR} ; \mathrm{sisq}=\mathrm{sigama}^{2}$;

dR

$\frac{4}{75}$

dtoR

$\frac{3}{640}$

sisq dtspatialsteps ${ }^{2}$

0.9

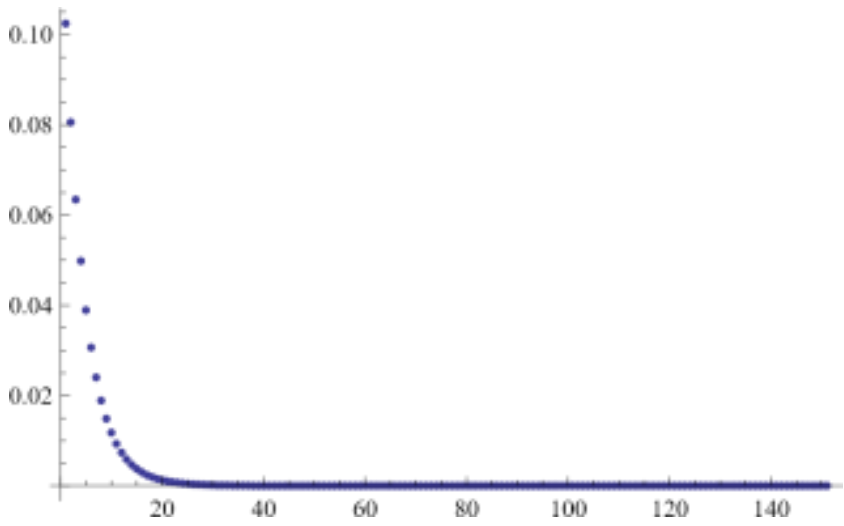

we connect the point and make the line smoothly, we get

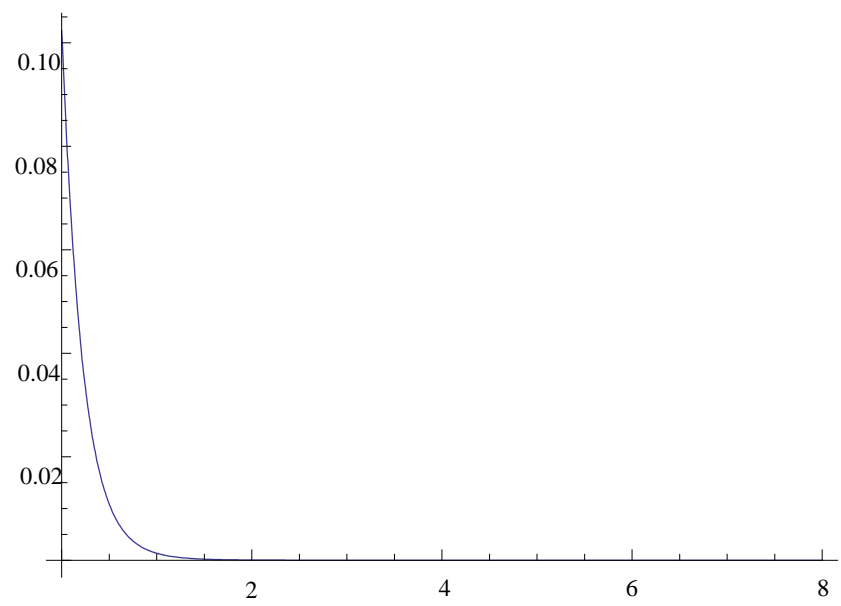

Finally, we get the value of $\mathrm{V}$, 


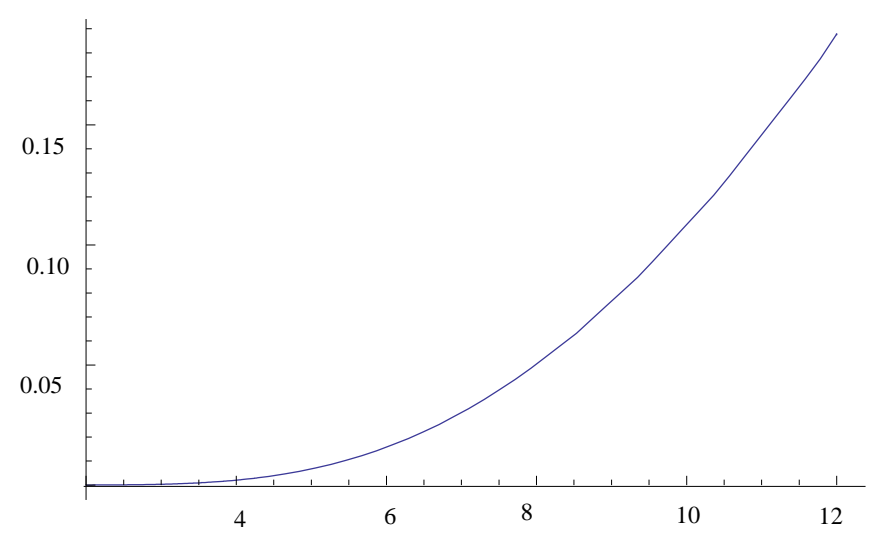

\section{Stability Analysis and Comparing}

we need execute some stability analysis, if we change the time steps value to 3000 and keep other parameter unchanged, we will see:

$\mathbf{r}=0.05 ;$ sigma $=0.4 ; \mathrm{T}=1 ; \operatorname{Rmax}=8 ;$

time steps = 3000; spatial steps $=150$;

dt $=\mathbf{T} /$ time steps; $\mathbf{d R}=\mathbf{R m a x} /$ spatial steps;

$\mathrm{dtoR}=\mathrm{dt} / \mathrm{dR} ; \operatorname{sisq}=\operatorname{sigma}^{2}$;

dR

$\frac{4}{75}$

dtoR

$\frac{1}{160}$

sisq dtspatialsteps ${ }^{2}$

1.2

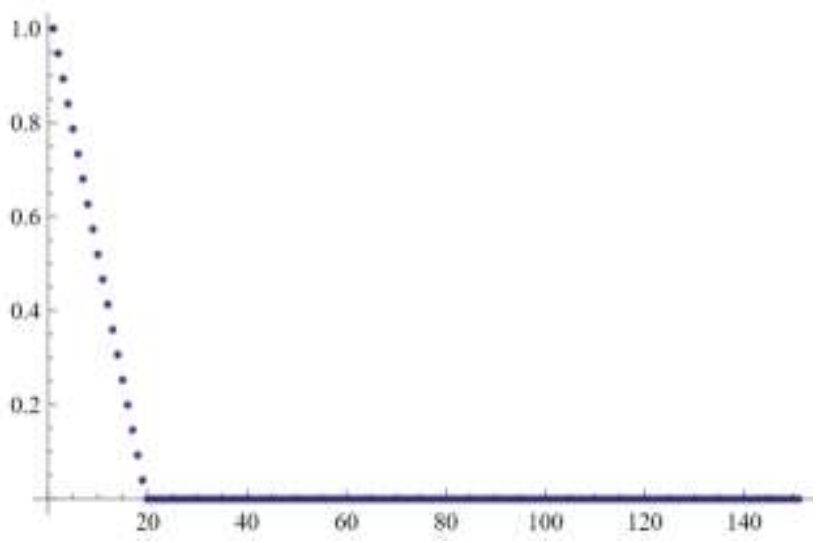

This picture shows h0 does not change. 


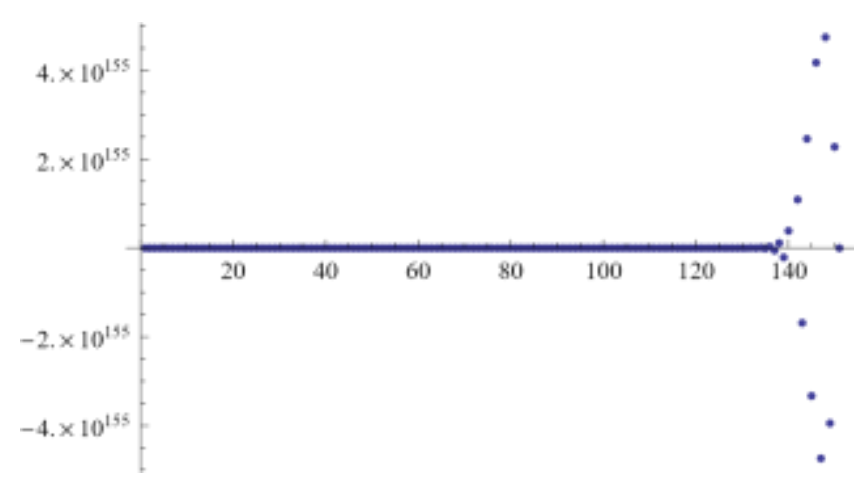

After we finished the recursive computation though, the picture completely becomes chaos.

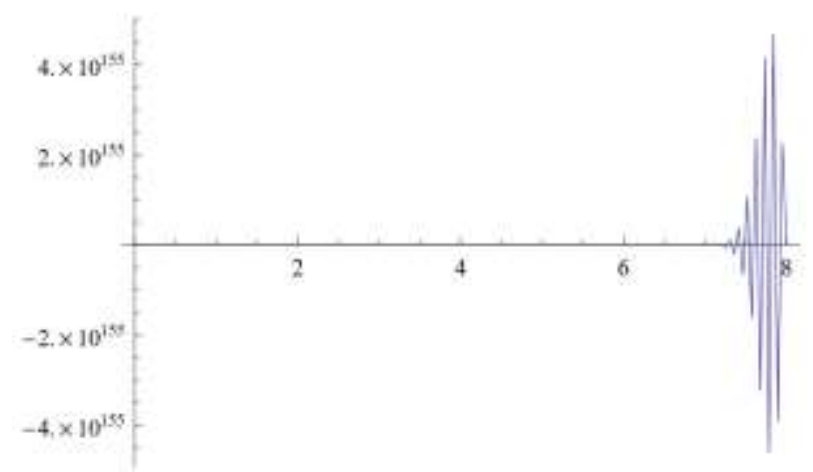

We connect the discrete points, we get the picture above. but the solution is useless.

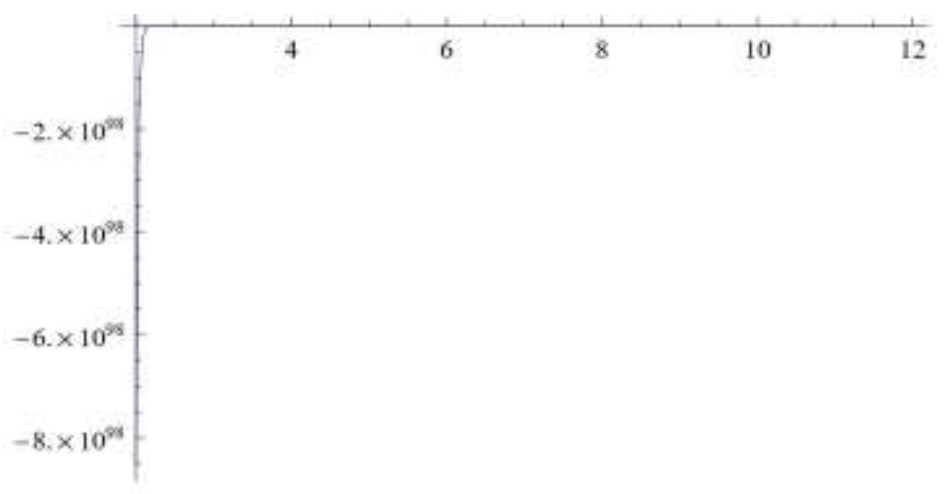

And the result is also bad.

Next step, we set

$\mathrm{r}=0.1, \sigma=0.4$ and $\mathrm{T}=0.5 \mathrm{Rmax}=1$.

timesteps $=\mathbf{5 0 0}$, spatialsteps $=\mathbf{5 0}$;

hnought $[R]:=\operatorname{Max}[1-R / T, 0.0]$

$\mathrm{r}=0.1 ;$ sigma $=0.4 ; \mathrm{T}=0.5 ; \operatorname{Rmax}=1$;

time steps $=500$; spatial steps $=50$;

$\mathrm{dt}=\mathrm{T} / \mathrm{time}$ steps; $\mathrm{dR}=\mathrm{Rmax} / \mathrm{spatial}$ steps; $\mathrm{dtoR}=\mathrm{dt} / \mathrm{dR} ; \mathrm{sisq}=\operatorname{sigma}^{2}$;

sisq dtspatialsteps ${ }^{2}$

0.4 


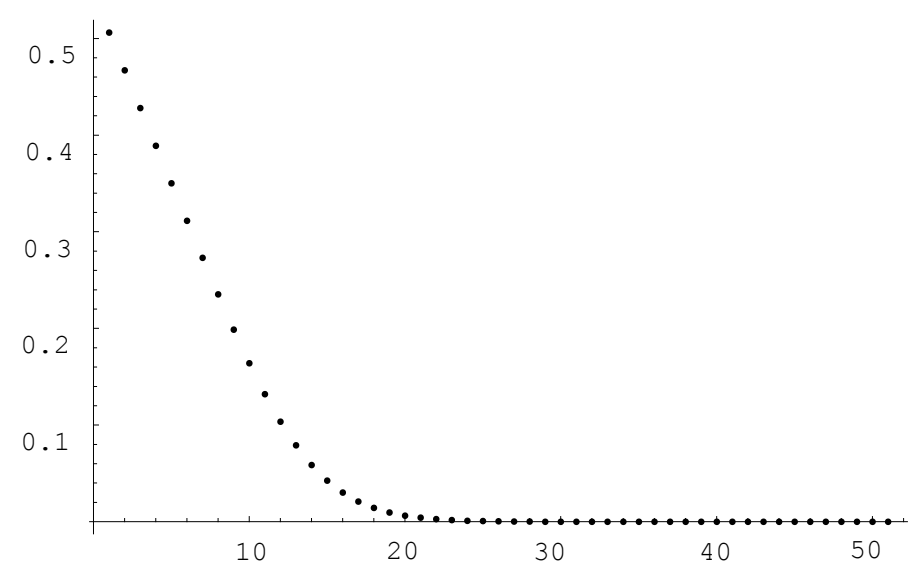

Connecting all the discrete point, we get:

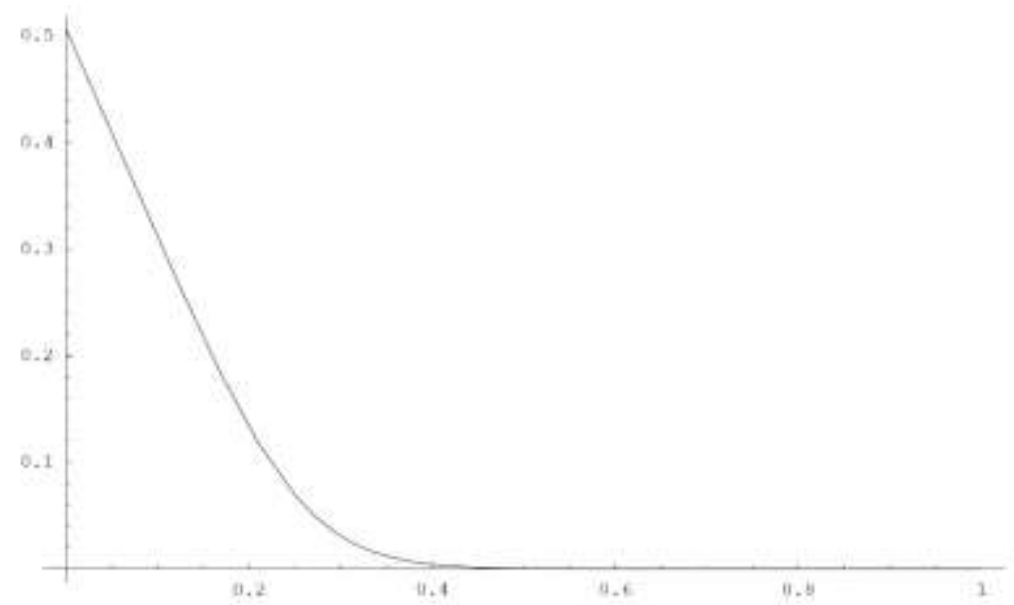

This group of parameter looks like no bad cause we choose fitted $\mathrm{dt} / \mathrm{dR}$, therefore we can conclude that the approximation accuracy has a close relationship with the value of $\mathrm{dt} / \mathrm{dR}$. In fact, if we choose bigger time steps and spatial steps, we can get more accurate result.

\section{Conclusions}

To the end, there are a variety of methods for pricing Asian options, some of which have been refereed in brief in this paper. As with all Numerical method pricing, the trade-off is between accuracy, speed and simplicity. Monte Carlo simulation is the simplest method for pricing Asian options, and it can be made arbitrarily accurate by increasing the number of simulations. Finite difference methods are much faster, but on the other hand, more complicated to implement. In addition, the choice of the PDE representation as well as the Finite Difference scheme to be used can affect the accuracy of the results achieved.

\section{Reference}

Alzairy, B., Decamps, J. P., \& Koehl, P. F. (1997). A P. D. E Approach to Pricing Asian Options: Analytical and Numerical Evidence, Journal of Banking and Finance, 21, 613-640. https://doi.org/10.1016/S0378-4266(96)00057-X

Barucci, E., Polidoro, S., \& Vespri, V. (2011). Some results on partial differential equations and asian options. Mathematical Models \& Methods in Applied Sciences, 11(3), 475-497. https://doi.org/10.1142/S0218202501000945

Cen, Z., Le, A., \& Xu, A. (2013). An alternating-direction implicit difference scheme for pricing asian options. Journal of Applied Mathematics, 2013(2), 627-648. https://doi.org/10.1155/2013/605943

Cen, Z., Le, A., \& Xu, A. (2013). Finite difference scheme with a moving mesh for pricing asian options. Applied Mathematics \& Computation, 219(16), 8667-8675. https://doi.org/10.1016/j.amc.2013.02.065

Cen, Z., Xu, A., \& Le, A. (2015). A hybrid finite difference scheme for pricing asian options. Applied Mathematics \& Computation, 252(C), 229-239. https://doi.org/10.1016/j.amc.2014.12.007 
D'Halluin, Y., Forsyth, P. A., \& Labahn, G. (2005). A semi-lagrangian approach for american asian options under jump diffusion. Siam Journal on Scientific Computing, 27(1), 315-345. https://doi.org/10.1137/030602630

Duffy, D. J. (2013). 23. Finite Difference Methods for Asian Options and Other 'Mixed' Problems. Finite Difference Methods in Financial Engineering: A Partial Differential Equation Approach. John Wiley \& Sons Ltd.

Francis, A. L., \& Eduardo, S. S. (2001). Valuing american options by simulation: a simple least-squares approach. Review of Financial Studies, 14(1), 113-147. https://doi.org/10.1093/rfs/14.1.113

Gerbessiotis, A. V. (2010). Parallel option price valuations with the explicit finite difference method. International Journal of Parallel Programming, 38(2), 159-182. https://doi.org/10.1007/s10766-009-0126-5

Goto, Y., Fei, Z., Kan, S., \& Kita, E. (2007). Options valuation by using radial basis function approximation. Engineering Analysis with Boundary Elements, 31(10), 836-843. https://doi.org/10.1016/j.enganabound.2007.02.001

Han, C. H. (2003). Pricing asian options with stochastic volatility. Quantitative Finance, 3(5), 353-362. https://doi.org/10.1088/1469-7688/3/5/301

Henderson, V., Hobson, D., Shaw, W., \& Wojakowski, R. (2003), Bounds for Floating Strike Asian Options using Symmetry.

Hugger, J. (2003). A fixed strike asian option and comments on its numerical solution. Anziam Journal, 2003(2003).

Kristoffer, G., Goran, P., \& Farman, S. (2010). The british asian option. Sequential Analysis, 29(3), 311-327. https://doi.org/10.1080/07474946.2010.487439

Kumar, A., Waikos, A., \& Chakrabarty, S. P. (2011). Pricing of average strike asian call option using numerical pde methods. International Journal of Pure \& Applied Mathematics, 76(5).

Li, Z. L., \& Lai, M. C. (2011). New finite difference methods based on iim for inextensible interfaces in incompressible flows. East Asian Journal on Applied Mathematics, 1(2), 155-171. https://doi.org/10.4208/eajam.030510.250910a

Lim, T. W. (2002). Performance of recursive integration for pricing european-style asian options. Preprint.

Meyer, G. H. (2001). On pricing american and asian options with pde methods. Acta Mathematica Universitatis Comenianae, 70(1), 2001.

Paul Wilmott, Sam Howison, Jeff Dewynne, The Mathematics of financial Derivatives, (University of Oxford and Imperial College, London, University of Oxford, University of Southampton)

Rogers, L., \& Shi, Z. (1995), The Value of an Asian Option, Journal of Applied Probability 32, 1077C1088.

Rudiger, U. S. Tools for Computational Finance, (University of K"oLn Institute of Mathematics Weyertal 86-90 50931 K"oLn, Germany)

Sak, H., Özekici, S., \& İlkay, B. (2007). Parallel computing in asian option pricing. Parallel Computing, 33(2), 92-108. https://doi.org/10.1016/j.parco.2006.11.002

Silva, A. J. D., Baczynski, J., \& Vicente, J. V. M. (2016). A new finite difference method for pricing and hedging fixed income derivatives: comparative analysis and the case of an asian option. Journal of Computational \& Applied Mathematics, 297, 98-116. https://doi.org/10.1016/j.cam.2015.10.025

Tan, X. (2013). A splitting method for fully nonlinear degenerate parabolic pdes. Electronic Journal of Probability, 18(18), 1-24. https://doi.org/10.1214/ejp.v18-1967

Umavathi, J. C., Kanth, A. S. V. R., \& Shekar, M. (2013). Comparison study of differential transform method with finite difference method for magnetoconvection in a vertical channel. Heat Transfer-Asian Research, 42(42), 243-258. https://doi.org/10.1002/htj.21035

Wu, L. X., Kwok, Y. K., \& Yu, H. (1999). Asian options with the american early exercise feature. International Journal of Theoretical \& Applied Finance, 1(1), 101--111. https://doi.org/10.1142/S021902499900008X

\section{Copyrights}

Copyright for this article is retained by the author(s), with first publication rights granted to the journal.

This is an open-access article distributed under the terms and conditions of the Creative Commons Attribution license which permits unrestricted use, distribution, and reproduction in any medium, provided the original work is properly cited. 